Check for updates

Cite this: RSC Adv., 2021, 11, 5444

Received 19th November 2020 Accepted 28th December 2020

DOI: 10.1039/d0ra09831k

rsc.li/rsc-advances

\section{Synthesis of thermally robust benzimidazolone- based wholly aromatic polyketones}

\author{
Xinming Gu, (D) ${ }^{a}$ Zhipeng Wang, ${ }^{\text {b }}$ Honghua Wang, ${ }^{\text {b }}$ Guangyuan Zhou ${ }^{\mathrm{b}}$ \\ and Yanmin Zhou*a
}

A series of wholly aromatic polyketones bearing benzimidazolone moieties (PK-BI) were synthesized via $\mathrm{N}-\mathrm{C}$ coupling polycondensation. Calcium carbonate coupled with potassium carbonate was used for the first time to achieve a high molecular weight, with $T_{\mathrm{g}}$ of the polymer as high as $299^{\circ} \mathrm{C}$. The polymer structure was confirmed by solid state ${ }^{13} \mathrm{C}$ NMR and FT-IR. The thermal stability of wholly aromatic polyketones with a benzimidazolone unit in the main chain was significantly improved, being higher than those of PEEKs and other amorphous PAEKs, proved by thermogravimetric analysis (TGA) and derivative thermogravimetric (DTG) analysis. The degradation activation energy $\left(E_{k}\right)$ values estimated by FlynnWall-Ozawa (FWO) and Kissinger methods were $260.33 \mathrm{~kJ} \mathrm{~mol}^{-1}$ and $282.57 \mathrm{~kJ} \mathrm{~mol}^{-1}$, respectively, which are higher than those of PEEKs.

\section{Introduction}

Aromatic polymers containing only aromatic rings and ketonic carbonyl groups as dominating linkages in the main chain are defined as wholly aromatic polyketones. ${ }^{1}$ The structural characteristics of the wholly aromatic polyketone endowed the polymer with longer conjugating units than poly(aryl ether ketone)s (PAEKs), which are an important class of high performance resins. In the main chain of PAEK, the ether bond was a weaker linkage compared to the carbonyl group. ${ }^{2}$ Therefore, for wholly aromatic polyketones, the absence of ether bonds in the main chain led to a more rigid structure, and the thermal stability was enhanced. Based on the above facts, it is a good idea to prepare wholly aromatic polyketones for better thermal stability, however, the synthesis in practice encountered some inconveniences.

The difficulty in synthesis of the wholly aromatic polyketones presumably led to the paucity of reports. The reason was suspected to be its rigid skeleton caused by an aromatic ring moiety and a carbonyl moiety aligning on the same plane, which accelerated the aggregation of oligomers and precipitated in the reaction solvents even at high temperature, resulting in the suppression of the molecular weight.

While some aliphatic polyketones ${ }^{3}$ had been synthesized, wholly aromatic polyketones, ${ }^{4-12}$ a new series of highperformance polymers, were reported by Yonezawa's group (see Scheme 1). There were some aliphatic substitutes such as

${ }^{a}$ Department of Oral Implantology, Hospital of Stomatology, Jilin University, Changchun 130021, China.E-mail: zhouym@jlu.edu.cn

${ }^{b}$ Division of Energy Materials (DNL22), Dalian Institute of Chemical Physics of the Chinese Academy of Sciences, Dalian 116023, China methoxyl and trifluoromethyl side groups in these polymers synthesized by either $\mathrm{C}-\mathrm{C}$ coupling polycondensation or electrophilic polycondensation considering the solubility of polymer. The glass transition temperatures $\left(T_{\mathrm{g}} \mathrm{s}\right)$ were usually lower than $220^{\circ} \mathrm{C}$. The methoxyl side groups in the polymer decreased the thermal stability of the polymer obviously compared with the aromatic backbone. Kricheldorf reported polyketones with the imidazolone unit and their poly ether ketone copolymers, but the research failed to get the wholly aromatic polyketone with benzimidazolone unit due to the low reactivity of $4,4^{\prime}$ diflorobenzophenone with silylated benzimidazolone in solid state melting polycondensation..$^{13}$ Allan reported a developed $\mathrm{N}-\mathrm{C}$ coupling polycondensation in TMS to synthesize the polysulfone with benzimidazolone unit. ${ }^{14}$ The polymer was easily soluble in chloroform and NMP.

Pyrolysis analysis was of importance in designing, processing and application of polymers. Systematic investigations
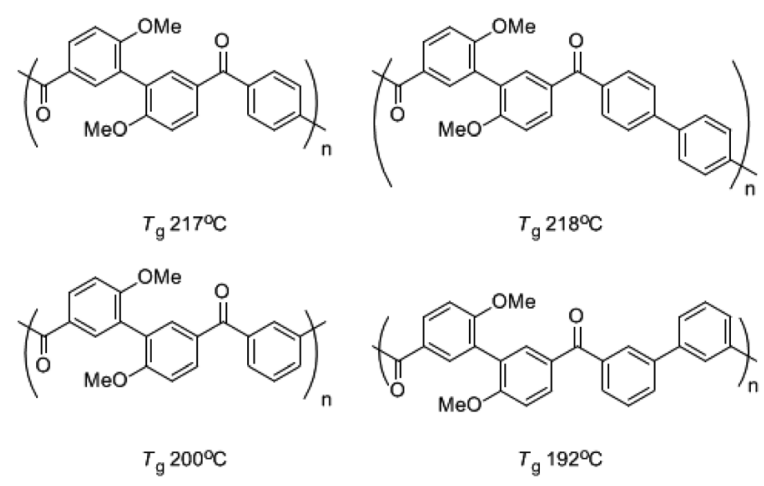

Scheme 1 Wholly aromatic polyketones. 
about polymer characterization and mechanisms provided an efficient method to improve the structure design and achieve higher thermal durability. Features of the aromatic polymer were depicted as follows: thermal stability increased with the relative number of the aromatic groups per repeat unit of the polymer chain. The pyrolysis tended to begin with the breakage of the weakest bond in the bridging groups between aromatic rings. The introduction of the heteroatoms such as nitrogen, phosphorus and silicon by homo- or copolymerization of the monomers tended to be stable, and raised the temperature of thermal pyrolysis.

The decomposition kinetics of aliphatic polyketones based on styrene and carbon monoxide was detailed investigated by Jia's group. ${ }^{15}$ The $E_{\mathrm{k}}$ values estimated via Flynn-Wall-Ozawa method and Kissinger method were found to be in the range from 270.72 to $297.55 \mathrm{~kJ} \mathrm{~mol}^{-1}$. The degradation kinetics of wholly aromatic polyketone was not systematically studied yet due to the difficulty to obtain the polymer.

Here, for the first time, we reported the synthesis and the thermal degradation of a new member of heat-resistant wholly aromatic polyketone with benzimidazolone unit, PK-BI. We presumed that the big size \& rigid benzimidazolone unit and nitrogen, as heteroatom, will both efficiently enhance the thermal durability compared to PEEK.

\section{Experimental}

\subsection{Materials and apparatus}

PK-BI (synthesized in our lab via $\mathrm{N}-\mathrm{C}$ coupling polycondensation, see Section 2.2), 1,3-dihydrobenzoimidazol-2one (1, from Alfa Aesar), 4,4'-difluorodiphenylmethanone (2, from Changzhou Huashan Chemical Co., Ltd, China), diphenylsulfone (DPS, purified prior to use), sulfolane (TMS, purified prior to use), 3-dimethyl-2-imidazolidinone (DMI, purified prior to use), 1-cyclohexyl-2-pyrrolidone (CHP, purified prior to use), anhydrous potassium carbonate and calcium carbonate (received from commercial sources and dried in vacuum at $120^{\circ} \mathrm{C}$ for $24 \mathrm{~h}$ ), poly ether ketone (PEEK, from Jilin University).

FT-IR spectrum was recorded on a Bio-Rad FTS-135 spectrophotometer. Solid state ${ }^{13} \mathrm{C}$ NMR $(100 \mathrm{MHz})$ spectrum was recorded on a Varian 400 instrument. Differential scanning calorimetry (DSC) was performed on a Mettler Toledo instrument DSC1 with a heating/cooling rate of $10 \mathrm{~K} \mathrm{~min}^{-1}$ in $\mathrm{N}_{2}$ (flow rate of $200 \mathrm{ml} \mathrm{min}^{-1}$ ). $T_{\mathrm{g}}$ was determined as the temperature at the midpoint of the thermal transition from the second heating curve. Thermogravimetric analysis (TGA) was performed on a Mettler Toledo instrument TGA/DSC1 at different heating rate from $5 \mathrm{~K} \mathrm{~min}^{-1}$ to $25 \mathrm{~K} \mathrm{~min}^{-1}$ under $\mathrm{N}_{2}$ or compressed air atmosphere (flow rate of $20 \mathrm{ml} \mathrm{min}^{-1}$ ) if the condition was not mentioned specially. $T_{-5 \%}$ was reported as the temperature at which $5 \%$ weight loss was observed.

\subsection{Synthesis of PK-BI}

Typical procedure of preparing the polymers was described as follows: a stoichiometric ratio of monomers with an excess amount of carbonate salts (mixture of $\mathrm{CaCO}_{3}$ and $\mathrm{K}_{2} \mathrm{CO}_{3}$ ) was dispersed in DPS. Other aprotic solvents such as DMI, CHP and TMS were also used for comparison in our experiments. Toluene was employed to azeotrope off the water at $135^{\circ} \mathrm{C}$ for $2 \mathrm{~h}$, and then distilled off. The reaction mixture was stirred at $200{ }^{\circ} \mathrm{C}$ for 8 to $12 \mathrm{~h}$ to obtain the solution of yellowish polymer. The mixture was diluted by DMAc when the temperature decreased to $150{ }^{\circ} \mathrm{C}$ naturally. The polymer precipitated in the acidified water and ethanol. The precipitation was washed with hot acetone in a Sohlex extractor to remove the solvent, then neutralized with acetic acid and washed in boiling water to remove the salts. The product was dried in vacuum at $120^{\circ} \mathrm{C}$ for $12 \mathrm{~h}$.

\subsection{Kinetic analysis of the decomposition process}

Thermogravimetric analysis (TGA) was the most common technique to study the thermal degradation property of specimen. For the kinetics analysis, it was assumed that a solid to gas phase transformation during the degradation process could be in accordance with the following formula:

$$
\frac{\mathrm{d} \alpha}{\mathrm{d} T}=k(T) f(\alpha)
$$

where $\alpha$ was the conversion degree defined as the ratio of the actual mass loss to the total mass loss, $k(T)$ was the reaction rate constant, $f(\alpha)$ was the kinetics model function that could take various mathematical forms depending on the physical mechanism..$^{16,17}$

When the reaction rate constant was expressed by the Arrhenius equation and taking $\beta$ as the heating rate, the reaction rate could be defined as eqn (2),

$$
\frac{\mathrm{d} \alpha}{\mathrm{d} T}=\left(\frac{A}{\beta}\right) \mathrm{e}^{-\left(E_{\mathrm{k}} / R T\right)} f(\alpha)
$$

2.3.1. Flynn-Wall-Ozawa method. Flynn-Wall-Ozawa method $(\mathrm{FWO})^{18}$ was a simple method to determine the apparent activation energy value directly from TGA curves. After separating the variables, integrating, eqn (2) becomes:

$$
g(\alpha)=\int_{0}^{\alpha} \frac{\mathrm{d} \alpha}{f(\alpha)}=\left(\frac{A}{\beta}\right) \int_{T_{0}}^{T} \mathrm{e}^{-\left(E_{\mathrm{k}} / R T\right)} \mathrm{d} T
$$

After integrating eqn (3) and taking logarithms, we obtained

$$
\lg \beta=\lg \frac{A E_{\mathrm{k}}}{\operatorname{Rg}(\alpha)}+\lg p(x)
$$

where $x=E_{\mathrm{k}} / R T, p(x)=\left(\frac{\mathrm{e}^{-1}}{x^{2}}\right) \sum_{n=1}(-1)^{n-1}\left(\frac{n !}{x^{n-1}}\right)$.

This function was based on the Doyle's approximation, when $20 \leq x \leq 60, \lg p(x)$ may be closely approximated by eqn (5),

$$
\lg p(x) \cong-2.135-0.457 \frac{E_{\mathrm{k}}}{R T}
$$

Then, eqn (4) became

$$
\lg \beta=\lg \frac{A E_{\mathrm{k}}}{R g(\alpha)}-2.135-0.457 \frac{E_{\mathrm{k}}}{R T}
$$


Therefore, the apparent activation energy $\left(E_{\mathrm{k}}\right)$ could be obtained from the plot of $\lg \beta$ against $1 / T$ for a fixed degree of conversion.

2.3.2. Kissinger method. Kissinger noticed the correlation between the peak temperature $\left(T_{\mathrm{p}}\right)$ and the heating rate $(\beta) .{ }^{19}$ If we assumed the order of reaction, then the kinetic function $f(\alpha)$ was shown as follows:

$$
f(\alpha)=(1-\alpha)^{n}
$$

where $n$ was the empirical reaction order. Substituting eqn (7) into eqn (2), we obtained

$$
\frac{\mathrm{d} \alpha}{\mathrm{d} T}=\left(\frac{A}{\beta}\right) \mathrm{e}^{-\left(E_{\mathrm{k}} / R T\right)}(1-\alpha)^{n}
$$

If the temperature raised at a constant rate $\beta$, then by differentiation of eqn (8) and defining that the maximum rate happened at the peak temperature $\left(T_{\mathrm{p}}\right)$, eqn (8) became

$$
g=\frac{E \mathrm{~d} T / \mathrm{d} t}{R T_{\mathrm{p}}{ }^{2}}=A n\left(1-\alpha_{\mathrm{p}}\right)^{n-1} \mathrm{e}^{-\left(E / R T_{\mathrm{p}}\right)}
$$

Kissinger pointed out the product $n(1-\alpha)^{n-1}$ was very nearly equal to unity. By substituting this value in eqn (9) and taking the logarithm on the both sides, we got

$$
\ln \left(\frac{\beta}{T_{\mathrm{p}}^{2}}\right)=\ln \frac{A_{\mathrm{k}} R}{E_{\mathrm{k}}}-\frac{E_{\mathrm{k}}}{R} \frac{1}{T_{\mathrm{p}}}
$$

It was possible for the determination of the degradation activation energy, $E_{\mathrm{k}}$, by eqn (10). The plot of $\ln \left(\beta / T_{\mathrm{p}}{ }^{2}\right) v s .1 / T_{\mathrm{p}}$ would be a straight line, the slope of which permitted the calculation of $E_{\mathrm{k}}$.

\subsection{Pyrolysis behaviour}

The pyrolysis behaviour of PK-BI was investigated on pyrolysisgas chromatography-mass spectrometry (Py-GC/MS) in helium (He) atmosphere at $500{ }^{\circ} \mathrm{C}, 850{ }^{\circ} \mathrm{C}$ and $1200^{\circ} \mathrm{C}$ respectively. The Py-GC/MS experiments were carried out using a CDS 5000 Pyroprobe pyrolyzer (Chemical Data System Co. Ltd) coupled to an HP 5890 gas chromatograph fitted with an Agilent 5975 mass spectrometer. The probe was calibrated by the manufacturer to ensure the accuracy of the nominally set temperatures. Sample was pyrolyzed at the setting temperature. The pyrolysis was

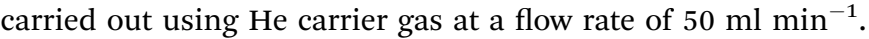
The GC column was an HP-1MS ( $30 \mathrm{~m}$ by $0.25 \mathrm{~mm}$ in diameter, $0.25 \mathrm{~mm}$ film thickness). Oven temperature was initially held for $3 \mathrm{~min}$ at $60{ }^{\circ} \mathrm{C}$, then programmed to $320^{\circ} \mathrm{C}$. The $\mathrm{GC} / \mathrm{MS}$ interface was set at $250{ }^{\circ} \mathrm{C}$. The flow rate was kept constant. The MSD was scanned from 20 to $300 \mathrm{~m} / \mathrm{z}$ at a data rate of $20 \mathrm{~Hz}$.

\section{Results and discussion}

\subsection{Synthesis and characterization of PK-BI}

Wholly aromatic polyketone with benzimidazolone moiety (PK$\mathrm{BI}$ ) was synthesized via the $\mathrm{N}-\mathrm{C}$ coupling polycondensation,

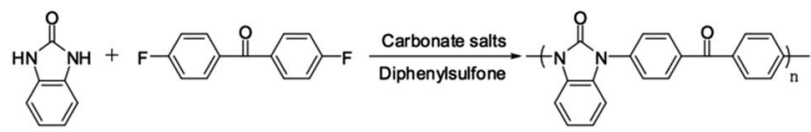

Scheme 2 Preparation of PK-BI.

Table 1 Reaction condition of wholly polyketone PK-BI containing imidazole moiety

\begin{tabular}{llllll} 
Entry & Solvent & Base & Temperature $/{ }^{\circ} \mathrm{C}$ & Time $/ \mathrm{h}$ & $T_{\mathrm{g}} /{ }^{\circ} \mathrm{C}$ \\
\hline 1 & $\mathrm{DMI}$ & $\mathrm{K}_{2} \mathrm{CO}_{3}$ & $129 / 188$ & $2 / 8$ & 238 \\
2 & $\mathrm{DMI}$ & $\mathrm{K}_{2} \mathrm{CO}_{3} / \mathrm{CaCO}_{3}$ & $129 / 188$ & $2 / 8$ & 244 \\
3 & $\mathrm{CHP}$ & $\mathrm{K}_{2} \mathrm{CO}_{3}$ & $136 / 208$ & $2 / 8$ & 243 \\
4 & $\mathrm{CHP}$ & $\mathrm{K}_{2} \mathrm{CO}_{3} / \mathrm{CaCO}_{3}$ & $135 / 208$ & $2 / 8$ & 251 \\
5 & $\mathrm{TMS}$ & $\mathrm{K}_{2} \mathrm{CO}_{3}$ & $135 / 200$ & $2 / 8$ & 229 \\
6 & $\mathrm{TMS}$ & $\mathrm{K}_{2} \mathrm{CO}_{3} / \mathrm{CaCO}_{3}$ & $135 / 200$ & $2 / 6$ & 234 \\
7 & $\mathrm{TMS}$ & $\mathrm{K}_{2} \mathrm{CO}_{3} / \mathrm{CaCO}_{3}$ & $135 / 200$ & $2 / 8$ & 239 \\
8 & $\mathrm{TMS}$ & $\mathrm{K}_{2} \mathrm{CO}_{3} / \mathrm{CaCO}_{3}$ & $135 / 200$ & $2 / 10$ & 242 \\
9 & $\mathrm{DPS}$ & $\mathrm{K}_{2} \mathrm{CO}_{3}$ & $135 / 200$ & $2 / 8$ & 273 \\
10 & $\mathrm{DPS}$ & $\mathrm{K}_{2} \mathrm{CO}_{3} / \mathrm{CaCO}_{3}$ & $135 / 190$ & $2 / 8$ & 265 \\
11 & $\mathrm{DPS}$ & $\mathrm{K}_{2} \mathrm{CO}_{3} / \mathrm{CaCO}_{3}$ & $135 / 195$ & $2 / 8$ & 275 \\
12 & $\mathrm{DPS}$ & $\mathrm{K}_{2} \mathrm{CO}_{3} / \mathrm{CaCO}_{3}$ & $135 / 200$ & $2 / 8$ & 299 \\
13 & DPS & $\mathrm{K}_{2} \mathrm{CO}_{3} / \mathrm{CaCO}_{3}$ & $135 / 200$ & $2 / 12$ & 299 \\
& & & & & \\
\hline
\end{tabular}

from 1,3-dihydrobenzoimidazol-2-one (1) and 4,4'-difluorodiphenylmethanone (2) in the presence of excess amount of carbonate salts in DPS solvent, or DMI, CHP and TMS instead, see Scheme 2. It was indispensable to use $\mathrm{CaCO}_{3}$ which accomplished the leave of the fluoride group in order to get the high molecular weight polymer (Table 1).

The effect of carbonate salts on the polymerization system was deeply investigated. The effect of calcium carbonate on the reaction could not be ignored. It was due to formation of insoluble inorganic product, calcium difluoride, with the aid of carbonate calcium. The polycondensation of monomers gave not only polymer PK-BI, but also the small molecules, such as water, carbon dioxide and potassium fluoride. With the presence of calcium carbonate, the fluoride ion would form the calcium fluoride and turn to precipitate, which lead to the accumulation of the reaction. It was revealed with the same result that a higher $T_{\mathrm{g}}$ was detected by the present of calcium salt than those without calcium salt among the four solvent DMI, CHP, TMS and DPS chosen in our experiment. We preferred DPS to other solvents including DMI, CHP and TMS in considering of the solubility of the polymer since the boiling point of DPS is high enough to ensure a high reactive temperature up to $200{ }^{\circ} \mathrm{C}$. The precipitated calcium fluoride could be removed by washing with acetic acid, which effectively improved the efficiency of polymerization.

The effects of time and temperature on the polymerization reaction were also obvious. With the increase of reaction time and temperature, the $T_{\mathrm{g}}$ of the polymer increased obviously. However, for DPS solvent system, entry 13, no increase for $T_{\mathrm{g}}$ was observed compared to entry 12 . This may be because the $T_{\mathrm{g}}$ increase was not obvious as the molecular weight of the polymer increased to a certain range, that is, when the molecular weight 


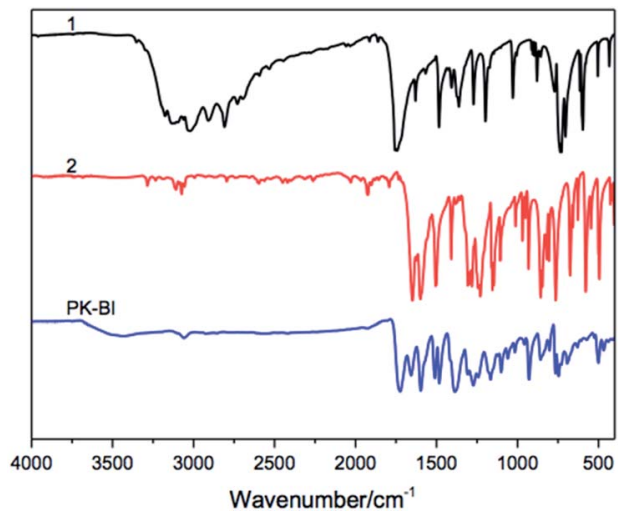

Fig. 1 FT-IR spectra of PK-BI and the monomers.

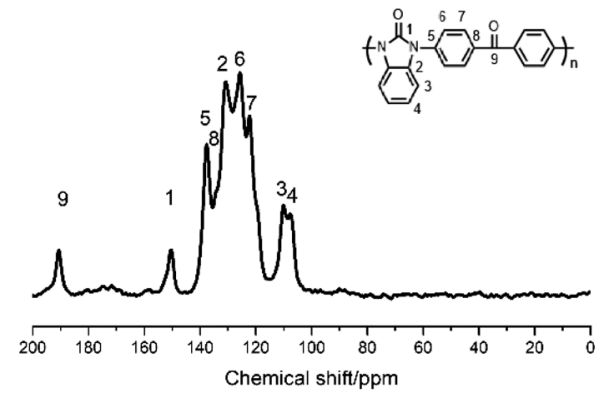

Fig. 2 Solid state ${ }^{13} \mathrm{C}$ NMR spectrum of PK-BI.

of the polymer reached a critical molecular weight, $T_{\mathrm{g}}$ tended to be a constant.

The structure of PK-BI prepared in DPS was characterized by the FT-IR (Fig. 1) and quantitative solid state ${ }^{13} \mathrm{C}$ NMR (Fig. 2) spectra. The FT-IR spectra of the polymer and the monomers thereof were shown in Fig. 1. The FT-IR spectrum of PK-BI indicated that $3059 \mathrm{~cm}^{-1}$ was attributed to the $\mathrm{C}-\mathrm{H}$ stretching vibration of the aromatic rings. The $\mathrm{C}=\mathrm{O}$ stretching vibration of the urea part occurred at $1724 \mathrm{~cm}^{-1}$ owing to the presence of the ring strain, and that of the diphenylmethanone unit occurred at $1657 \mathrm{~cm}^{-1}, 1386 \mathrm{~cm}^{-1}$ and $1165 \mathrm{~cm}^{-1}$ were attributed to the asymmetric and symmetric stretching vibration of the $\mathrm{N}-\mathrm{C}-\mathrm{N}$ group respectively.

Solid state ${ }^{13} \mathrm{C}$ NMR was an effective method to characterize the structure of chemicals which were insoluble in common solvents. In Fig. 2, the signals of $190.68 \mathrm{ppm}$ and $150.39 \mathrm{ppm}$ were assigned to the C9 of diphenylmethanone unit and C1 of benzimidazolone moiety respectively. They were the critical peaks in the polymer. The solid state ${ }^{13} \mathrm{C}$ NMR spectrum combined with the FT-IR spectrum confirmed that the structure was in accordance with that we designed.

Thermal property was evaluated by differential scanning calorimetry (DSC) and the second heating curve was shown in Fig. 3. There was only one glass transition at $299^{\circ} \mathrm{C}$. No melting transition was found during the test temperature range. It was in accordance with the theoretical results of the Allan S. Hay's research. The barrier to rotation of the $\mathrm{C}-\mathrm{O}-\mathrm{C}$ bond in the

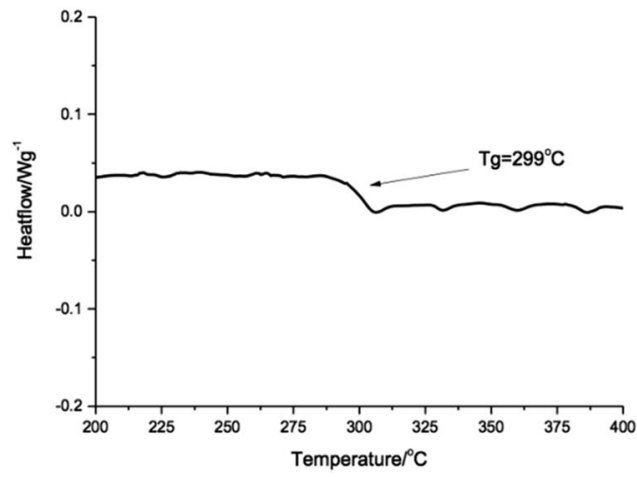

Fig. 3 The second heating DSC thermogram of PK-BI.

poly(aryl ether) resin was only $2.17 \mathrm{kcal} \mathrm{mol}^{-1}$, whereas the barrier to rotation of the $\mathrm{C}-\mathrm{N}-\mathrm{C}$ bond was calculated to be $41.79 \mathrm{kcal} \mathrm{mol}^{-1}$. The reason was that the conjugating $o$-phenylene pendent in the main polymer chain inhabited the ordered packing of the polymer. The derivative of imidazolidone unit was superior in respect to its distorting abilities, which suppressed the aggregation of the resulting polymer and maintained its solubility at the reaction temperature in DPS during the polymerization. It was necessary to note that the polymer prepared in TMS in the same condition gave a $T_{\mathrm{g}}$ of $239^{\circ} \mathrm{C}$, which was $60^{\circ} \mathrm{C}$ lower than that of the polymer prepared above. This was because that the solubility of PK-BI in TMS was so poor that the oligomer would precipitate when the toluene was distilled off. The lower molecular weight of the polymer would dramatically debase the thermal and mechanical properties of the polymer. The reaction indicated that DPS was a favorable solvent in preparing polymer PK-BI.

\subsection{Thermal degradation study}

Thermal stability of PK-BI was measured by TGA. The $T_{-5 \%}$, $T_{\text {onset }}$ and $T_{\mathrm{p}}$ were determined, see Table 2. PK-BI with aromatic rings exhibited good ablation resistant property with the char yield higher than $65 \%$ at $800{ }^{\circ} \mathrm{C}$ in $\mathrm{N}_{2}$. It must be stressed that PK-BI showed better thermal stability than PEEK, a typical polymer in special engineering plastics at present. The probable reason for the better thermal stability was the elimination of ether linkage and the existing of C-N bond linkage in the PK-BI backbone. The bond energy of $\mathrm{C}-\mathrm{N}$ was much higher than that of $\mathrm{C}-\mathrm{O}$ (ether) bond.

PK-BI was studied by the two methods above to unveil the kinetic of the pyrogenation. The Ozawa method was reliable and

Table 2 Thermal stability of PK-BI measured at different heating rate

\begin{tabular}{lllll}
\hline Samples & $\beta / \mathrm{K} \mathrm{min}^{-1}$ & $T_{-5 \%} /{ }^{\circ} \mathrm{C}$ & $T_{\text {onset }} /{ }^{\circ} \mathrm{C}$ & $T_{\mathrm{p}} /{ }^{\circ} \mathrm{C}$ \\
\hline PK-BI & 5 & 566 & 554 & 575 \\
PK-BI & 10 & 578 & 569 & 591 \\
PK-BI & 15 & 588 & 580 & 603 \\
PK-BI & 20 & 594 & 587 & 610 \\
PK-BI & 25 & 600 & 593 & 617 \\
PEEK & 10 & 546 & 547 & 569
\end{tabular}



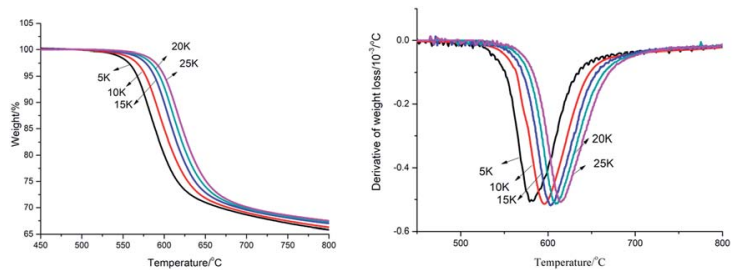

Fig. 4 TGA and DTG curves of PK-BI with different heating rate.

suitable for the analysis of polymers without knowing the reaction mechanism. ${ }^{19}$ Different heating rate of PK-BI was tested. The results of heating rate at $5 \mathrm{~K} \mathrm{~min}^{-1}, 10 \mathrm{~K} \mathrm{~min}^{-1}, 15$ $\mathrm{K} \mathrm{m^{-1 }}, 20 \mathrm{~K} \mathrm{~min}^{-1}$ and $25 \mathrm{~K} \mathrm{~min}^{-1}$ were charted in Fig. 4. Since the weight loss percentage of PK-BI was higher than $65 \%$, the activation energy was calculated from weight loss $5 \%$ to $30 \%$.

The temperature at which the polymer weight loss happened at a certain heating rate was listed in Table 3. The apparent activation energy was calculated by the slope of $\lg \beta-1 / T$ at different heating rates of 10,15 and $20 \mathrm{~K} \mathrm{~min}^{-1}$ according to eqn (6). The average of the apparent activation energy was $260.33 \mathrm{~kJ} \mathrm{~mol}^{-1}$ which was much higher than that of PEEK calculated by the same method $\left(231.8 \mathrm{~kJ} \mathrm{~mol}^{-1}\right)$.

The Kissinger method was described as eqn (10). Thermal analysis of DTG showed the dynamic process when the polymer was heated. The rate of weight loss vs. temperature was determined. The activation energy (see Table 4) calculated by the Kissinger method from the thermogravimetric data of DTG was $282.57 \mathrm{~kJ} \mathrm{~mol}^{-1}$, which was higher than that calculated from FWO method.

The difference between the FWO and Kissinger methods was $22.24 \mathrm{~kJ} \mathrm{~mol}^{-1}$, owing to the high char yield even at the final temperature. Most of the residues which remained by the complex mechanism were nonvolatile.

The TGA and DTG curves of PK-BI in $\mathrm{N}_{2}$ or compressed air at a heating rate of $10 \mathrm{~K} \mathrm{~min}^{-1}$ were depicted in Fig. 5, and those of PEEK in $\mathrm{N}_{2}$ atmosphere were given for control. In $\mathrm{N}_{2}$, the $T_{-5 \%}$ and $T_{\mathrm{p}}$ of PK-BI were $32{ }^{\circ} \mathrm{C}$ and $25^{\circ} \mathrm{C}$ higher than those of PEEK respectively. The reason was that the present of heteroatom instead of ether linkage in PK-BI had higher bond energy than that of PEEK. The char yield values of PK-BI and PEEK in $\mathrm{N}_{2}$ at $900{ }^{\circ} \mathrm{C}$ were $62.0 \%$ and $52.6 \%$. The residues of PK-BI contained more nonvolatile chemicals. The decomposition results of PK$\mathrm{BI}$ in $\mathrm{N}_{2}$ and compressed air atmosphere were similar with
Table 4 Thermal parameters and activation energies obtained from FWO and Kissinger method

\begin{tabular}{lllll}
\hline & & & \multicolumn{2}{l}{$E_{\mathrm{k}}\left(\mathrm{kJ} \mathrm{mol}^{-1}\right)$} \\
\cline { 5 - 5 }$\beta\left(\mathrm{K} \mathrm{min}^{-1}\right)$ & $T_{\mathrm{P}}(\mathrm{K})$ & $1000 / T\left(\mathrm{~K}^{-1}\right)$ & FWO & Kissinger \\
\hline 10 & 864 & 1.157 & 260.33 & 282.57 \\
15 & 876 & 1.141 & & \\
20 & 883 & 1.132 & &
\end{tabular}

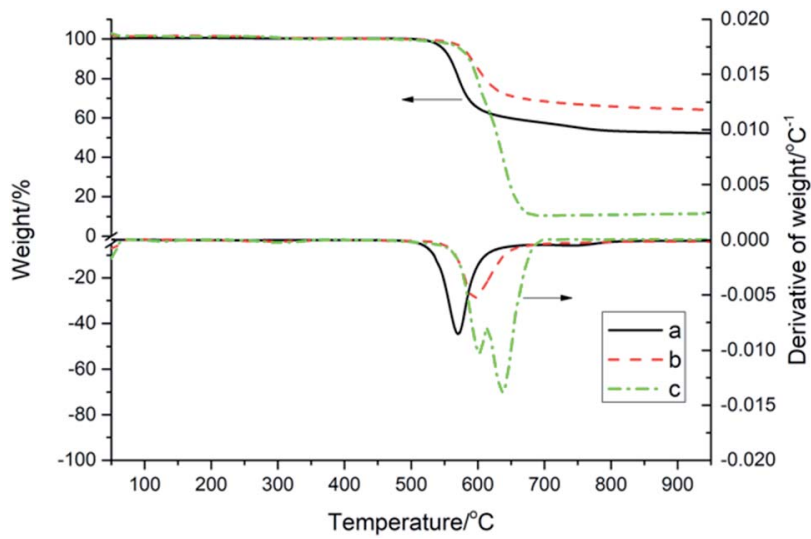

Fig. 5 TGA and DTG curves of PK-BI and PEEK at a heating rate of 10 $\mathrm{K}_{\text {min }}^{-1}$ : (a) PEEK in $\mathrm{N}_{2}$; (b) PK-BI in $\mathrm{N}_{2}$; (c) PK-BI in compressed air.

each other when the temperature was below $550{ }^{\circ} \mathrm{C}$. When the temperature increased, one peak was found in the DTG curve in $\mathrm{N}_{2}$ and the number for compressed air was two. The reason was that the oxygen in air reacted with the splitting aromatic free radicals. Oxygenation of aromatic rings took place and the volatile chemicals accounted for the large amount of weight loss. In compressed air, the rate of weight loss at $590{ }^{\circ} \mathrm{C}$ was twice of that in $\mathrm{N}_{2}$. The second peak was found at $644^{\circ} \mathrm{C}$. Much more volatile chemicals after oxygenation made the further weight loss. $68.6 \%$ of weight loss was found from $600{ }^{\circ} \mathrm{C}$ to $690{ }^{\circ} \mathrm{C}$. The weight of nonvolatile degradation chemicals was $6.3 \%$ at $900{ }^{\circ} \mathrm{C}$.

\subsection{Py-GC/MS analysis}

Py-GC/MS was used to discuss the volatile segments decomposed of PK-BI at $500{ }^{\circ} \mathrm{C}, 850{ }^{\circ} \mathrm{C}$, and $1200{ }^{\circ} \mathrm{C}$, see Table 5 and Fig. 6.

Table 3 Thermal degradation active energies of $\mathrm{PK}-\mathrm{BI}$ at different weight loss

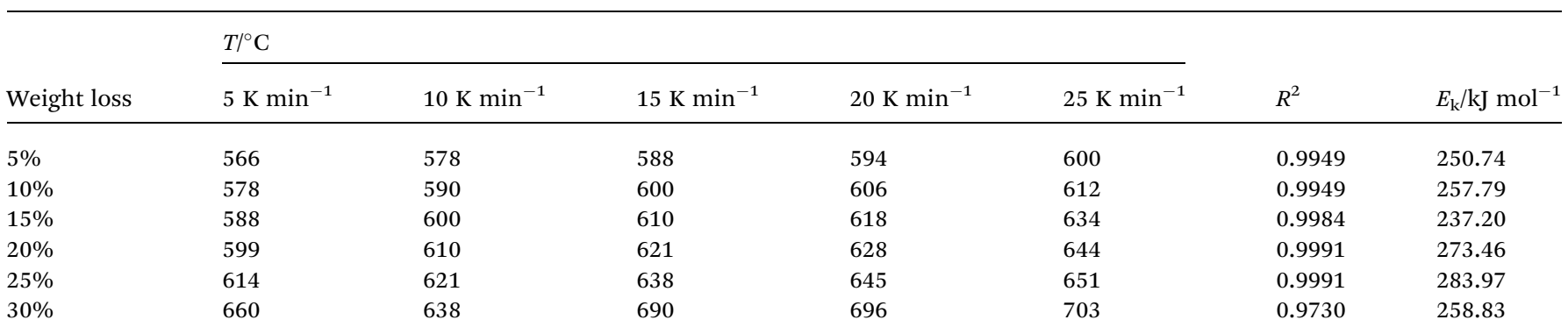


Table 5 Thermal degradation products of $\mathrm{PK}-\mathrm{BI}$ in He atmosphere

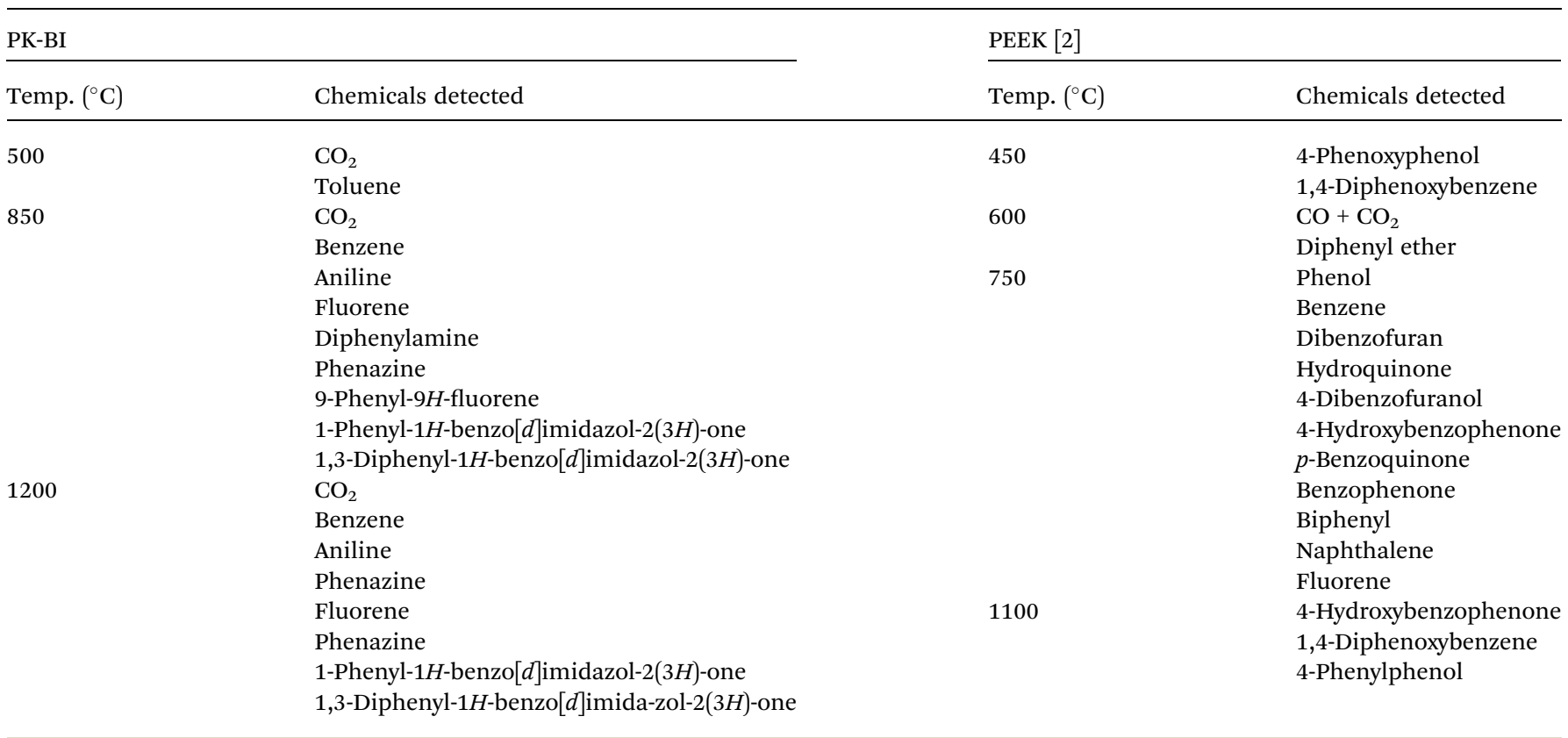

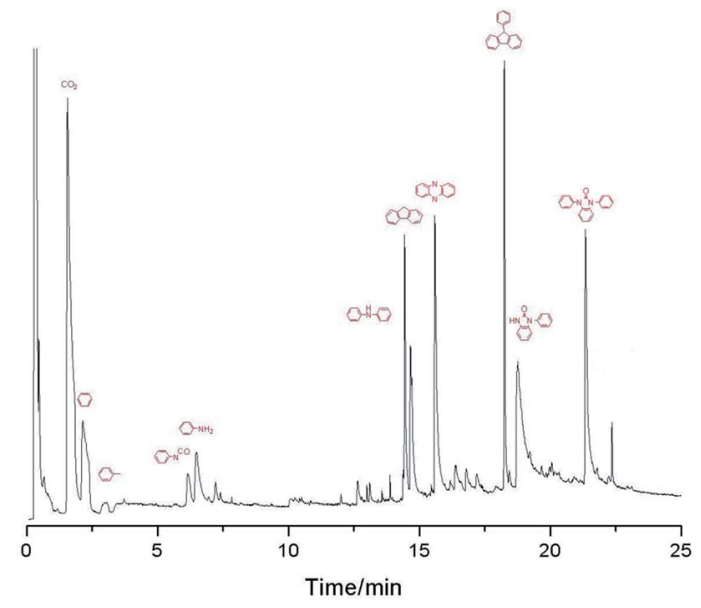

Fig. 6 Py-GC-MS result of PK-BI at temperature of $850^{\circ} \mathrm{C}$.

The results indicated that the organic decomposition chemicals were mainly the segments containing heteroatom aromatic organics and the aromatic ring such as aniline, fluorine and 9-phenyl-9H-fluorene. The volatile products at $500{ }^{\circ} \mathrm{C}$ were mainly carbon dioxide and toluene, indicating that the none conjugated ketonic carbonyl group was the weak bond when heated. With the test temperature ascending, 1-phenylbenzimidazolone accounting for large amounts of volatile products was detected which provided an indirect hint that the formation of $\mathrm{C}-\mathrm{N}$ linkage in the reaction. The Py-GC/MS result was similar with that of PEEK ${ }^{8,20}$ except the heteroatom introduced by the benzimidazolone unit.

\section{Conclusions}

Wholly aromatic polyketones containing rigid benzimidazolone unit was successfully synthesized via $\mathrm{N}-\mathrm{C}$ coupling polycondensation. Calcium carbonate as part of the catalysts, was used for the first time to achieve a high molecular weight, with $T_{\mathrm{g}}$ of the polymer as high as $299^{\circ} \mathrm{C}$. The polymer was confirmed to be highly heat-resistant due to the introducing of steric benzimidazolone unit via thermogravimetric analysis. The apparent activation energy of the polymer calculated by the Flynn-WallOzawa method and Kissinger method was found to be higher than those of PEEKs which were the commercial special engineering plastics. The Py-GC/MS tests at different temperature indicated that the non-conjugated ketonic carbonyl group acted as the weaker bond in the main polymer chain than $\mathrm{N}-\mathrm{C}$ bond when decomposition happened. The research results showed that the benzimidazolone-based aromatic polyketones remarkably enhanced the thermal stability. This prospected that the polymer had potential application in the aerospace, aviation and other fields as a super heat-resistant material.

\section{Conflicts of interest}

The authors declare no conflict of interest.

\section{Acknowledgements}

The authors thank the National Science Foundation of China (No. 51703220), Innovative Foundation of Dalian Institute of Chemical Physics (No. DICP I202033), Department of Finance of Jilin Province (jcsz2020304-1) and Department of Science and Technology of Jilin Province (20200201302JC) for the financial support.

\section{References}

1 J. Yang and H. W. Gibson, Macromolecules, 1997, 30, 56295633. 
2 P. Patel, T. R. Hull, R. W. McCabe, D. Flath, J. Grasmeder and M. Percy, Polym. Degrad. Stab., 2010, 95, 709-718.

3 W. Fan, J. Mu, S. Shan, H. Su, S. Wu and Q. Jia, Catal. Commun., 2014, 49, 34-38.

4 N. Yonezawa, H. Nakamura and K. Maeyama, React. Funct. Polym., 2002, 52, 19-30.

5 N. Yonezawa, S. Mori, S. Miyata, Y. Ueha-Anyashiki, S. M. Wu and K. Maeyama, Polym. J., 2003, 35, 998-1002.

6 K. Maeyama, Y. Tagata, H. Nishimori, M. Yamazaki, S. Maruyama and N. Yonezawa, React. Funct. Polym., 2004, 61, 71-79.

7 K. Maeyama, S. Maeda, H. Saito and N. Yonezawa, Polym. J., 2007, 39, 342-346.

8 N. Yonezawa, S. Miyata, T. Nakamura, S. Mori, Y. Ueha and R. Katakai, Macromolecules, 1993, 26, 5262-5263.

9 L. H. Perng, C. J. Tsai and Y. C. Ling, Polymer, 1999, 40, 73217329.

10 J. L. Yang, C. S. Tyberg and H. W. Gibson, Macromolecules, 1999, 32, 8259-8268.
11 F. C. Rix, M. Brookhart and P. S. White, J. Am. Chem. Soc., 1996, 118, 4746-4764.

12 Y. Zhang, X. Sun, Y. Niu, G. Wang and Z. Jiang, Synth. Met., 2006, 156, 893-897.

13 H. R. Kricheldorf and O. Stöber, Eur. Polym. J., 1992, 28, 1377-1382.

14 A. A. Mir, S. Matsumura, A. R. Hlil and A. S. Hay, ACS Macro Lett., 2012, 1, 194-197.

15 J. Mu, W. Fan, S. Shan, H. Su, S. Wu and Q. Jia, Thermochim. Acta, 2014, 579, 74-79.

16 B. Jankovic, Chem. Eng. J., 2008, 139, 128-135.

17 S. Vyazovkin, Model-free kinetics, J. Therm. Anal. Calorim., 2006, 83, 45-51.

18 J. H. Flynn and L. A. Wall, J. Polym. Sci., Part B: Polym. Lett., 1966, 4, 323-328.

19 H. E. Kissinger, Anal. Chem., 1957, 29, 1702-1706.

20 A. Ramgobin, G. Fontaine and S. Bourbigot, Polymers, 2020, 12, 1789. 